\title{
A literature review on the conflict analysis of vehicle-pedestrian interactions
}

\author{
A. Kizawi ${ }^{1, *}$, A. Borsos ${ }^{1}$ \\ ${ }^{1}$ Széchenyi István University, Department of Transport Infrastructure and Water \\ Resources Engineering \\ Egyetem tér 1., 9026 Győr, Hungary \\ *e-mail: ahmad.kizawi.24@gmail.com
}

Submitted: 05/03/2021; Accepted: 23/03/2021; Published online: 08/04/2021

\begin{abstract}
An alternative to traffic safety analysis based on historical crash data the use of non-crash events is becoming more popular thanks to the rapid improvement in video-based vehicle trajectory processing. By means of Surrogate Measures of Safety (SMoS) in traffic conflict studies, the most critical elements on the road network can be identified and the probability of accidents can be proactively determined. This paper aims to summarize the state-of-the-art research regarding the analysis of pedestrian-vehicle interactions at unsignalized crossings, to synthetize the previous studies using Surrogate Measures of Safety $(\mathrm{SMoS})$, and to identify the research gaps.
\end{abstract}

Keywords: traffic conflict; Surrogate Measures of Safety (SMoS); road safety; pedestrian

\section{Introduction}

The consequences of road accidents represent a real problem worldwide. Despite of the implementation many different effective traffic measures in the EU, the number of road accidents remains unacceptably high. The paper by Fülep and Óberling [1] provided the causal factors of road accidents with the appropriate recommendations. Since the statistical database are easily available (from Hungary and Europe), accident issues can be recognized: approximately 1.3 million crashes annually lead to nearly 40,000 fatalities and more than 1 million injuries. 
Several comprehensive studies in the European Union discuss road accidents from both analytical and statistical perspectives. The paper by Holló [26] provided an overview about road safety in the EU and Hungary including some statistical studies. Although there was no significant increase in the number of motor vehicles between 1987 and 1990, the number of road accident deaths in the EU increased significantly. However, from 2001 until 2009, overall the number of fatal victims of road accidents lessened by $36 \%$ in the EU member states thanks to the planned development by the European Union transport policy. [26]

The most sensible method for road safety assessment is scrutinizing the accidents using data records. However, this kind of methods has some restrictions especially for Vulnerable Road Users (VRUs) such as bicyclists and pedestrians. These limitations are related to underreporting problems, and missing accident data. From an ethical point of view, it makes no sense to wait for road accidents to occur to evaluate safety which we primarily want to avoid. [2]

Various researchers have developed a number of safety indicators aiming to overcome the shortcomings of safety analyses based on accidents (underreporting, quality issues and rare nature). By using Surrogate Measures of Safety (SMoS), the probability of accident at a location can be proactively determined before it would happen. There is a consensus among researches that observable non-crash situations can be useful for traffic safety assessment as a substitutional tool in parallel with analysis based on crash data [3], [4].

This paper aims to synthetize the previous studies using Surrogate Measures of Safety (SMoS) and to illustrate the most important indicators that can be used in traffic studies to evaluate the traffic conflicts seriousness.

\section{Literature review}

\subsection{Pedestrian-vehicle interactions}

Pedestrians and vehicles share the road in the complex internal traffic of the city resulting in interactions between vehicles and pedestrians affecting each other's movement. While vehicle trajectories are more predictable, there are several issues regarding pedestrian movements since pedestrians often tend to make more sudden decisions and more prone to hectically change their speed and trajectory. For pedestrian and vehicle interactions at intersections, the traffic conflict between the pedestrian and the vehicle is an important factor that influences some intersection characteristics such as capacity and safety. Conflicts between pedestrians and vehicles are growing in developing countries due to the ever-increasing traffic. The frequent interactions between vehicles and pedestrians deserve special interest to analyze safety at intersections. [5] 
The priority of the pedestrians at the unsignalized crosswalks is not entirely clear. This leads to higher levels of accident of pedestrians compared to signalized crosswalks. Pedestrian and Vehicle Interactions (PVI) have been receiving more attention among governmental agencies and decision-makers aiming to improve road accident countermeasures that decrease the rate of accidents and severe conflicts [6].

The study by Dey and Terken [7] attempted to define the importance of communication between drivers and pedestrians. Their study was carried out at a city in the Netherlands, at an intersection where approximately a hundred observations about pedestrian actions were observed at the pedestrian crossing after video recordings and field measurements for pedestrian's movements. At the pedestrian crossing, five behaviors were detected from pedestrians while interacting with approaching vehicles. Most of pedestrians (61\%) stepped on the pedestrian crossing without waiting for the driver's approval, while in some cases (20.7\%), pedestrians acted by changing their path based on a vehicle's approach. In other cases $(12.6 \%)$, pedestrians wait until an oncoming vehicle decelerates, and then they stepped on the pedestrian crossing. In $1.8 \%$ of the cases, a pedestrian tended to give the driver the priority for passing first. In $3.6 \%$ of the cases, pedestrians wait for an approaching car to completely stop, and get the driver's approval to cross.

\subsection{Traffic conflict analysis}

The first study to evolve a measure by which road accidents can be predicted was designed by Perkins and Harris [8]. Their study could be useful to gain a better insight into causal factors regarding traffic safety issues. In their study, they identified different potential accident situations, which they classified as traffic conflicts. They clarified more than twenty types of traffic conflicts that occur between road users, which were essentially identified by the occurrence of evasive actions, such as swerving, stopping, and braking.

In the terms of traffic conflicts based on evasive actions, the study by Johnsson et al. [9] described different surrogate measures of safety in the literature. Few indicators concentrated on aspects that can be useful when studying Vulnerable Road Users (VRUs) issues. Their paper evaluated different safety indicators depending on the capability of these indicators to take into account both injury risk and collision risk (talking the evasive actions into account). The findings in their paper showed that several indicators have focused on braking as an important indicator to define the critical traffic situations and do not consider other kinds of evasive actions such as running or swerving.

The supposition that there is a relationship between the seriousness and the frequency of traffic events is the basic idea behind the use of non-crash situations to 
investigate road safety [10]. Hyden [11] clarified the so-called "safety pyramid" where the upper part represents the most severe events, which can be considered as the rarest events in traffic, that we generally define them as "accidents". Immediately below the accidents come traffic conflicts, which are grouped as severe, slight or potential conflicts according to their dangerousness. Below the conflicts come the majority of traffic encounters that are characterized as natural events. Fig. 1 shows the Safety pyramid with the severity levels of traffic events.

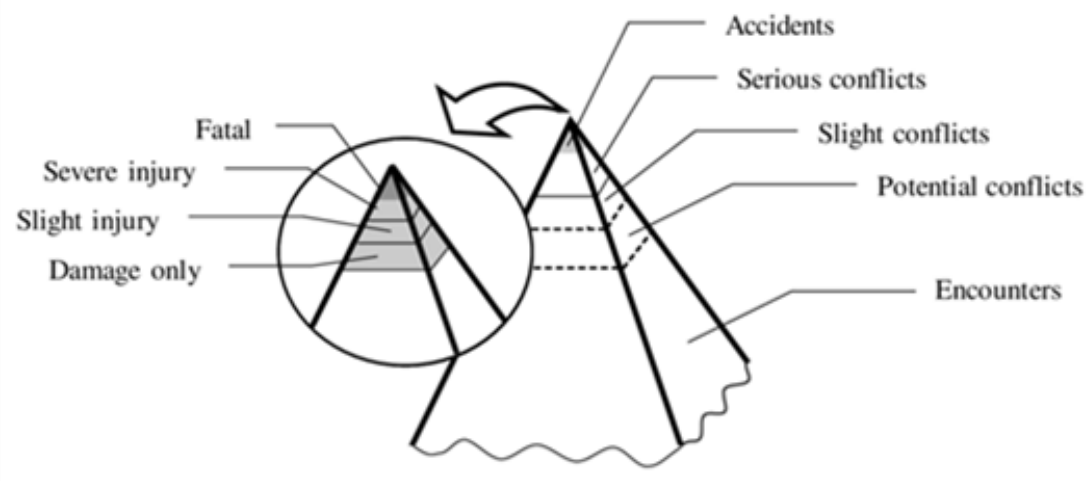

Figure 1. "Safety pyramid" [11]

\subsection{Surrogate Measures of Safety (SMoS)}

The term "surrogate" reveals that the indicators do not depend on crash database. Instead, they are meant to be complementary tools of historical records analysis. Several different indicators were proposed and developed by various researchers throughout the 1970s and the 1980s. Recently, several traffic safety indicators have been proposed and developed including those related to VRUs. Since there are many issues related to VRUs safety analysis such as underreporting problems of VRU crashes, there is a growing awareness in transport modes including VRUs [9].

Several papers have described, summarized and compared a group of safety indicators. For instance, Laureshyn et al. [4], [12] provided an overview about nearness to-collision and severity indicators. Zheng et al. [13] clarified the temporal and spatial proximity characteristics in traffic situations. An observed situation is considered a traffic conflict or not depending on closeness in distance and (or) time of the concerned road users.

Ceunynck [3] presented the previous studies on the application of safety indicators and looked into the frequency of use. He compiled indicators into groups using the Time-To-Collision (TTC), the Post Encroachment Time (PET), and the deceleration 
families, plus two extra groups for other and unspecified indicators. According to the study by Ceunynck [3], indicators originating from the (TTC) family are frequently used, followed by those from the (PET) family. There are a few indicators that do not belong to these families and are classified as "other" type, as shown in Fig. 2.

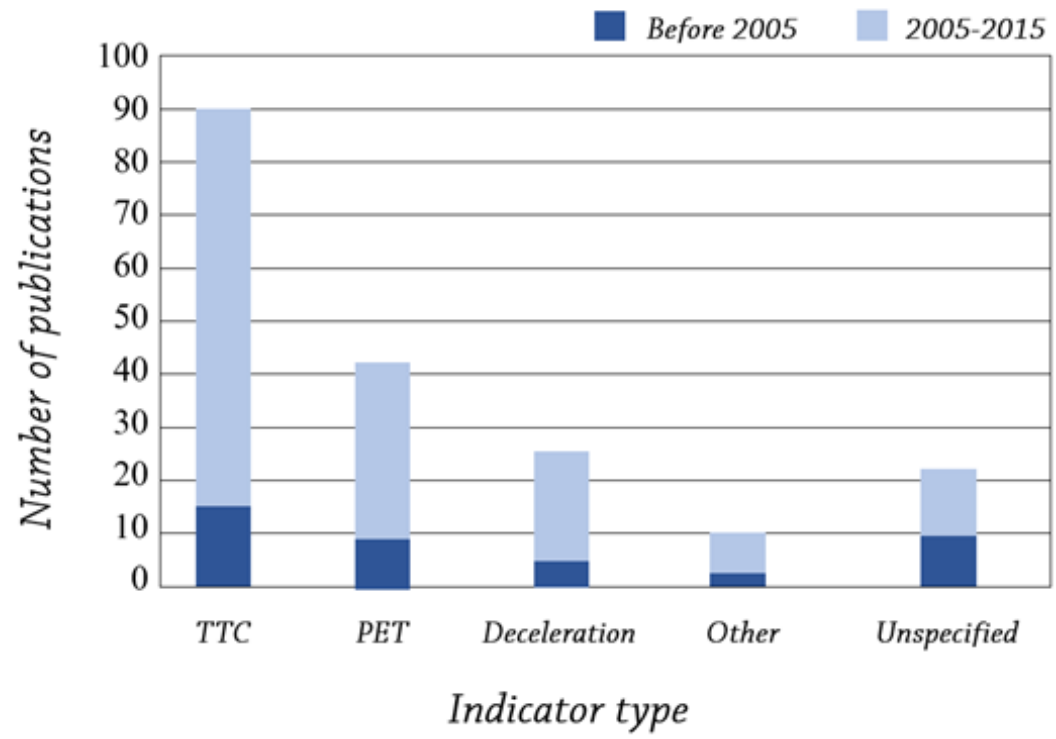

Figure 2. Frequency of using surrogate safety indicators [3]

The "unspecified" type belongs to papers that present incomplete details to determine the techniques that have been adopted.

The most commonly used Surrogate Measures of Safety (SMoSs) for pedestrian conflict analysis include but not limited to the indicators described in the following subsections. [14] These will be illustrated using figures showing vehicle-vehicle interactions; however, their calculation is identical to vehicle pedestrian interactions. 


\subsubsection{Time-To-Collision (TTC)}

This indicator has been suggested by Hayward [15]. It is the time to collision in seconds when the two vehicles continue their trajectory at the same angle and at the same speeds without any kind of evasive behaviors. The minimum value is 0 second (which means collision). When TTC has a small value, there is a high risk of collision.

Fig. 3 illustrates TTC for right-angle collision. In this case, TTC is calculated by the following equation (1), (2).

$$
\begin{aligned}
& \text { TTC }=\frac{d_{2}}{v_{2}}, \text { if } \frac{d_{1}}{v_{1}}<\frac{d_{2}}{v_{2}}<\frac{d_{1}+l_{1}+w_{2}}{v_{1}}, \\
& \text { TTC }=\frac{d_{1}}{v_{1}}, \text { if } \frac{d_{2}}{v_{2}}<\frac{d_{1}}{v_{1}}<\frac{d_{2}+l_{2}+w_{1}}{v_{2}},
\end{aligned}
$$

Where $v_{1}$ and $v_{2}$ are vehicles speeds; $d_{1}$ and $d_{2}$ are distances from the front of the vehicle to the conflict area; $l_{1}, l_{2}$, and $w_{1}, w_{2}$ are lengths and widths of vehicles, respectively.

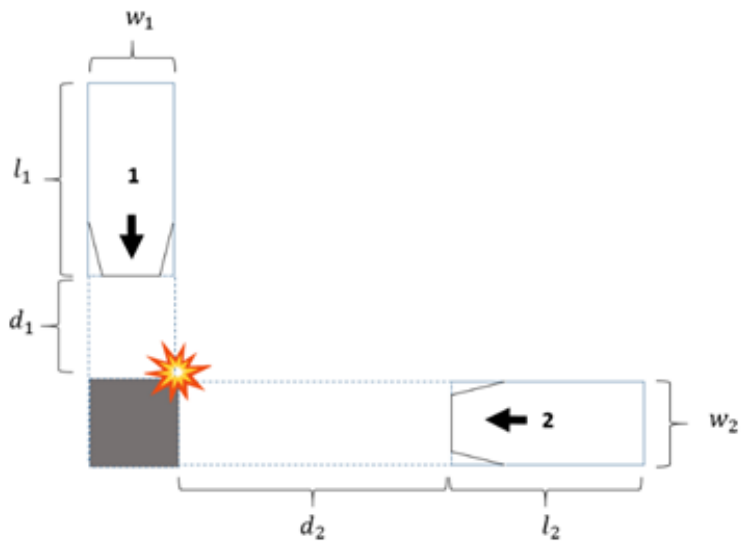

Figure 3. Illustration of TTC [17]

There are two important indicators that can be used based on TTC: Time to Accident (TA), which is the TTC at the moment in which an involved road user acts by making an evasive action, and TTC min, which is the minimum TTC value calculated in a conflict. For differentiating between a serious and a not serious event, these two indicators usually use a threshold value. 
Jiang [16] conducted a study about the vehicle-pedestrian conflict by field observations, video recordings, and TTC calculations. According to the paper, 3.0 and $1.0 \mathrm{~s}$ can be recommended for urban areas as the average TTC and TTCmin, respectively.

\subsubsection{Post Encroachment Time (PET)}

It is the difference between times when a car enters a conflict point until another one arrives to this conflict point [17], [18]. When PET has a small value, this indicates a higher dangerous of vehicle- pedestrian collisions.

To measure PET in the case of pedestrian-vehicle conflict, we only need to measure the passing times at the conflict point for both conflicting road users, while to measure TTC, we need to determine the time remaining to the conflict point at each time instant. Fig. 4 displays the procedure for calculating PET.
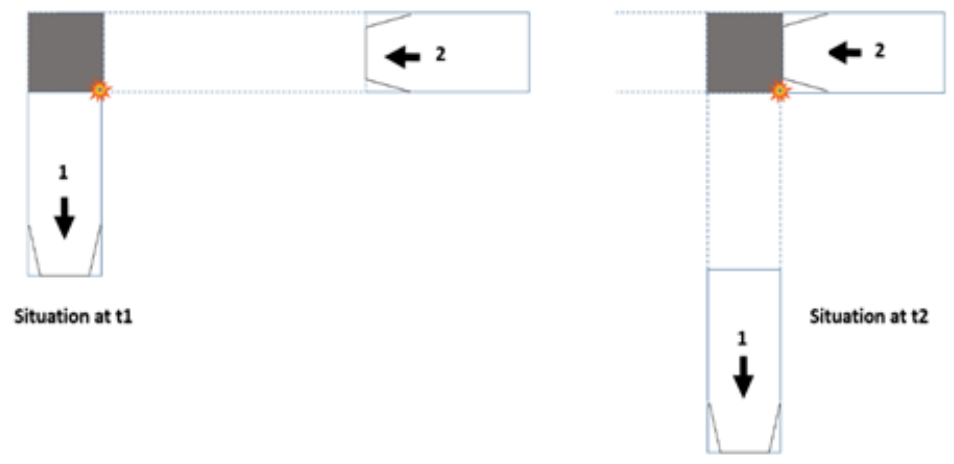

$$
\text { PET }=\mathbf{t} \mathbf{2}-\mathbf{t} \mathbf{1}
$$

Figure 4. Illustration of PET [17]

Several papers [17] [19] [20] found that PET and TTC indicators are considered as the most precise indicators for road safety assessment at intersections due to the simplicity of mensuration, consistency over time, and its relationship to other indicators. 


\subsubsection{Delta V}

This indicator reveals the velocity vector change experienced by the involved road user during an accident. It is very susceptible to the vulnerability of the road user, since a light body will bounce back when colliding with a massive one, while the speed of the massive body will not change. This is an important measure in accidents studies between, for example, a truck and a bicyclist. [3]

For an inelastic collision, Delta V values are calculated for each of the two conflicting road users using the following equation (3),(4). The highest value is used to describe severity. Fig. 5 illustrates the Delta V calculation based on the principle of momentum conservation.

$$
\begin{aligned}
& \Delta v_{1}=\frac{m_{2}}{m_{1}+m_{2}} \cdot \sqrt{v_{1}^{2}+v_{2}^{2}-2 v_{1} v_{2} \cos \alpha}, \\
& \Delta v_{2}=\frac{m_{1}}{m_{1}+m_{2}} \cdot \sqrt{v_{1}^{2}+v_{2}^{2}-2 v_{1} v_{2} \cos \alpha},
\end{aligned}
$$

Where: $v_{1}, v_{2}$ the speeds of the involved road users 1 and 2 respectively; $m_{1}, m_{2}$ their masses; $\alpha$ : the approach angle.
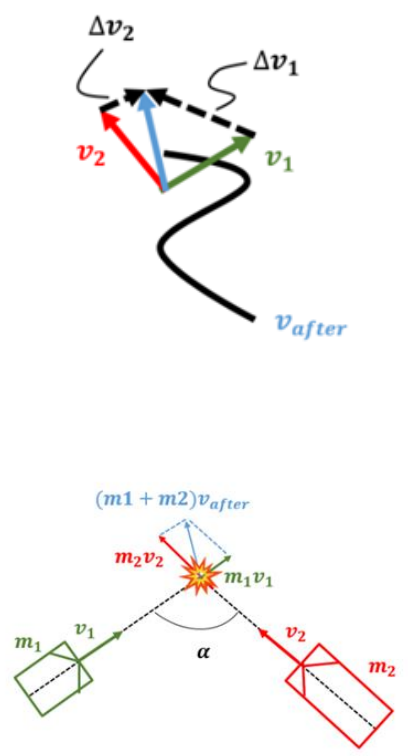

Figure 5. Calculation of Delta V [3] 


\subsection{Using extreme value theory (EVT) in traffic conflicts}

Extreme Value Theory (EVT) is useful for estimating crash probabilities using Surrogate Measures of Safety (SMoS). To evaluate the benefits of active safety techniques, the Weibull type of generalized extreme value (GEV) distribution was applied to traffic events [21]. The EVT method was also used and validated in [2]. For estimating the frequency of right-angle collisions at intersections, the GEV distribution was further used and the findings concluded that there was a favorable relationship between the estimated accidents and detected accidents. Tarko [22] clarified the use of the generalized Pareto distribution (GPD) as a complementary approach of GEV distribution in the EVT method, especially for estimating the severity of crashes based on traffic conflicts.

Some studies [23] used the EVT method in order to evaluate the head-on conflicts associated with crossing maneuvers situations in rural areas on two-lane roadways. Other researches [24] adopted the EVT method for estimating the road departure accident recurrence based on the index of time to road edge passing. It was found plausible accident estimates compared to the recorded accident database.

A recent study [25] aimed to anticipate the near-accident situations that can happen at signalized intersections between vehicles and pedestrians. With TTC and PET indicators obtained from field measurements and video recording, EVT method was applied to fit the distributions of PET and TTCmin values and to extract the threshold values. A Gated Recurrent Unit (GRU) neural network was used to for anticipating the most severe situations after the sequent data was obtained from pedestrians' and vehicles' movements, with an accuracy of 0.878 and the AUC (Area Under the Curve) value of 0.865 .The model presented in the study is considered as a useful model to alert drivers of the potential severe traffic situations with the pedestrians.

\section{Research gap and methods}

As for pedestrian-vehicle interactions, there are still some gaps related to the use of Surrogate Measures of Safety (SMoS) especially since the vulnerable road users (VRUs) tend to make sudden changes in their trajectories. The analysis of such interactions using Extreme Value Theory is also very scarce in the currently available literature.

The research related to the current paper focuses on analyzing pedestrian-vehicle interactions at unsignalized crossings using Surrogate Measures of Safety (SMoS) and Extreme Value Theory (EVT). An important characteristic of the EVT is that it enables the researcher to model the stochastic behavior of unusually large or small processes. This behavior is typically unobservable within a plausible data collection time period due to its rareness. [27] The assumption behind the EVT method is that 
the underlying stochastic behavior of the process being modeled is sufficiently smooth to enable extrapolations to unobserved levels [28]. EVT involves estimating extreme events probability over an extended period of time given very short and limited historical data. [27]

According to Tarko et al. [29] The Extreme Value Method offers an important advantage over the traffic conflict technique that the risk of crash given the surrogate event is estimated for any conditions based on the observed variability of crash proximity without using crash data. The crash proximity measure precisely defines the surrogate event. An unsignalized intersection in the city of Györ, Hungary has been selected where several different pedestrian-vehicle interactions will be video recorded. Video recordings will be used to collect SMoS with the help of the TAnalyst Software. EVT approach will be used to determine the probability of crashes and the ability of SMoSs to assess the severity of pedestrian-vehicle interactions. After the in-depth analysis, recommendations will be suggested for improving the levels of safety.

\section{Conclusions}

In this paper, we gave an overview about using Surrogate Measures of Safety $(\mathrm{SMoS})$ and the latest research results with regard to the analysis of pedestrianvehicle conflicts. The most important indicators that can be used to evaluate the seriousness of such conflicts such as TTC, PET and Delta-V were introduced. Extreme Value Theory has been recently used by a few researchers to estimate crash probabilities using SMoS; the most relevant literature sources in relation to that were summarized. It is concluded that there is an existing research gap in the conflict analysis of pedestrian-vehicle conflicts mainly because of the less predictable behavior of pedestrian movements. The use of SMoSs coupled with EVT to such conflicts also offers research opportunities.

\section{References}

[1] T. Fülep, J. Óberling, Traffic Safety based on Accident Statistics Concerning Road Vehicles and Infrastructure, Acta Technica Jaurinensis 5 (3) (2012) pp. 197-205.

doi: https://doi.org/10.14513/actatechjaur.v12.n1.489

[2] P. Songchitruksa, A. P. Tarko., The extreme value theory approach to safety estimation, Accident Analysis \& Prevention 38 (4) (2006) pp. 811-822.

doi: https://doi.org/10.1016/j.aap.2006.02.003 
[3] D. Ceunynck, Defining and applying surrogate safety measures and behavioural indicators through site-based observations Ph.D. thesis, Lund University (2017). [cited 2021-03-05]

URL

https://portal.research.lu.se/portal/files/30184385/17082 3Dissertation_TimDeCeunynck_final_inclcover.pdf

[4] Laureshyn A., Svensson Å., Hydén, C., Evaluation of traffic safety, based on micro-level behavioral data: Theoretical framework and first implementation. Accident Analysis \& Prevention 42 (6) (2010) pp. 1637-1646. doi: https://doi.org/10.1016/j.aap.2010.03.021

[5] A. Kumar, M. Paul, I. Ghosh, Analysis of pedestrian conflict with right turning vehicles at signalized intersections in India, Proactive safety assessment and improvements at intersections, Journal of Transportation Engineering Part (A) Systems 145 (6) (2018) pp. pp. 04019018-0104019018-12.

doi: https://doi.org/10.1061/JTEPBS.0000239

[6] H. Amado, S. Ferreira et al., Pedestrian-vehicle interaction at unsignalized crosswalks, A systematic review. Sustainability 12 (7) (2020) pp. 1-23. doi: https://doi.org/10.3390/su12072805

[7] D. Dey, J. Terken, Pedestrian interaction with vehicles, Roles of explicit and implicit communication, in: Automotive UI 2017 - 9th International ACM Conference on Automotive User Interfaces and Interactive Vehicular Applications, Proceedings, Association for Computing Machinery, Oldenburg, 2017, pp. 109-113.

doi: https://doi.org/10.1145/3122986.3123009

[8] R. S. Perkins, J. I. Harris, Traffic conflict characteristics-Accident potential at intersections, Highway Research Record 225 (1968) pp. 35-43.

[9] C. Johnsson, A. Laureshyn, T. De Ceunynck, In search of surrogate safety indicators for vulnerable road users, a review of surrogate safety indicators, Transport Reviews 38 (6) (2018) pp. 765-785.

doi: https://doi.org/10.1080/01441647.2018.1442888

[10] H. C. Chin, S. T. Quek, Measurement of traffic conflicts, Safety Science 26 (3) (1997) pp. 169-185. 
[11] C. Hydén, The development of a method for traffic safety evaluation, the Swedish traffic conflict technique, Doctoral thesis, Lund University, Department of Traffic Planning and Engineering (1987).

[12] A. Laureshyn, T. De Ceunynck et al., In search of the severity dimension of traffic events, Extended Delta-V as a traffic conflict indicator, Accident Analysis \& Prevention 98 (2017) pp. 46-56.

doi: https://doi.org/10.1016/j.aap.2016.09.026

[13] L. Zheng, K. Ismail, X. Meng, Traffic conflict techniques for road safety analysis, Open questions and some insights, Canadian Journal of Civil Engineering 41 (7) (2014) pp. 633-641.

doi: https://doi.org/10.1139/cjce-2013-0558

[14] P. Chen, W. Zeng et al., Surrogate Safety Analysis of Pedestrian-Vehicle Conflict at Intersections Using Unmanned Aerial Vehicle Videos. Journal of Advanced Transportation, (2017).

doi: https://doi.org/10.1155/2017/5202150

[15] J. C. Hayward, Near-miss determination through use of a scale of danger, Highway Research Record 384 (1972) pp. 24-34.

[16] X. Jiang, Intercultural Analyses of Time-to Collision in Vehicle Pedestrian Conflict on an Urban Midblock Crosswalk, IEEE transactions on intelligent transportation systems 16 (2) (2015) pp. 1-6.

doi: https://doi.org/10.1109/tits.2014.2345555

[17] B. L. Allen, B. T. Shin, D. J. Cooper, Analysis of traffic conflicts and collision, Transportation Research Record 667(1978) pp. 67-74.

[18] P. J. Cooper, Experience with traffic conflicts in Canada with emphasis on "post encroachment time" techniques, In Proceedings of the NATO Advanced Research Workshop on International Calibration Study of Traffic Conflict Technique (1983).

[19] D. Gettman, L. Head, Surrogate Safety Measures from Traffic Simulation Models, Final Report, Publication No. FHWARD-03-050, Federal Highway Administration, Washington, DC, USA. (2003). 
[20] D. Gettman, L. Sayed et al., Surrogate Safety Assessment Model and Validation," FHWA Report, Publication No.: FHWA-HRT-08-051, Federal Highway Administration, Washington, DC, USA. (2008).

[21] K. Campbell, H. C. Joksch, P. E. Green, A bridging analysis for estimating the benefis of active safety technologies, UMTRI-96-18, Final Report. University of Michigan, Transportation Research Institute (1996).

[22] A. P. Tarko, Use of crash surrogates and exceedance statistics to estimate road safety, Accident Analysis \& Prevention 45 (2012) pp. 230-240. doi: https://doi.org/10.1016/j.aap.2011.07.008

[23] H. Farah, C. L. Azevdo, Safety analysis of passing maneuvers using extreme value theory, IATSS Research 41 (1) (2017) pp. 12-21. doi: https://doi.org/10.1016/j.iatssr.2016.07.001

[24] T. J. Gordon, A Multivariate Analysis of Crash and Naturalistic Driving Data in Relation to Highway Factors Report No. S2-S01C-RW-1. Transportation Research Board, Washington, DC (2013).

[25] S. Zhang, M. Abdel-Aty et al., Modeling pedestrians' near-accident events at signalized intersections using gated recurrent unit (GRU), Accident Analysis \& Prevention 148 (2020) 105844. doi: https://doi.org/10.1016/j.aap.2020.105844

[26] P. Holló, Road Safety Situation of Hungary Reflected by National and International Objectives, Acta Technica Jaurinensis 4 (2) (2011) pp. 2-7.

[27] P. Songchitruksa, A. P. Tarko, The extreme value theory approach to safety estimation. Accident Analysis and Prevention (2006), 38:811-822.

[28] S. Coles, An Introduction to Statistical Modeling of Extreme Values, (2001)

[29] A. Tarko, G. Davis, N. Saunier, T. Sayed, S. Washington, White Paper SURROGATE MEASURES OF SAFETY ANB20(3) Subcommittee on Surrogate Measures of Safety ANB20 Committee on Safety Data Evaluation and Analysis (2009). 